\title{
Agradecimento
}

\section{Agradecimento aos Revisores da Revista Brasileira de Cardiologia Invasiva}

Revista Brasileira de Cardiologia Invasiva agradece aos membros do Conselho Editorial e aos revisores convidados abaixo listados, que compartilharam sua expertise e conhecimento científico, possibilitando a publicação de artigos de alto nível científico, dentro do mais absoluto rigor ético:

\section{Revisores:}

Alexandre Schaan de Quadros (RS)

André Labrunie (PR)

Ari Mandil (MG)

Carlos Antonio Mascia Gottschall (RS)

Carlos Augusto Cardoso Pedra (SP)

Cláudia Jacob Chaves (EUA)

Claudia Maria Rodrigues Alves (SP)

Edgar Guimarães Victor (PE)

Edson Antonio Bregagnollo (SP)

Eduardo Missel (PR)

Expedito Eustáquio Ribeiro da Silva (SP)

Fábio Sândoli de Brito Júnior (SP)

Fernando Stuchi Devito (SP)

Francisco José Araújo Chamié (RJ)

George Cesar Ximenes Meireles (SP)

Gilberto Lahorgue Nunes (RS)

Gilson Soares Feitosa (BA)

Hélio Roque Figueira (RJ)

João Paulo Zouvi (RS)
Jorge Pinto Ribeiro (RS)

José Antonio Marin-Neto (SP)

José Armando Mangione (SP)

José de Ribamar Costa Jr. (SP)

José Klauber Roger Carneiro (CE)

Luís Henrique Wolff Gowdak (SP)

Luiz Alberto Mattos (SP)

Marcelo Antônio Cartaxo Queiroga Lopes (PB)

Marcelo de Freitas Santos (PR)

Marco Vugman Wainstein (RS)

Marinella Centemero (SP)

Maurício Rezende Barbosa (MG)

Paulo Ricardo Avancini Caramori (RS)

Pedro Alves Lemos Neto (SP)

Ricardo Alves da Costa (SP)

Rodrigo Bellio de Mattos Barretto (SP)

Rogério Sarmento-Leite (RS)

Sergio Braga (SP)

Valmir Fernandes Fontes (SP)

Valter Lima (SP) 